\title{
Isothermal Titration Calorimetry as a Method for Analyzing Protein Adsorption in Ion Exchange Chromatography
}

\author{
Joao SIMOES-CARDoso $^{1}$, Nanako HoshinO ${ }^{1}$, Noriko YoSHIMOTO $^{1}$, and Shuichi YAMAMOTO ${ }^{*}$ \\ ${ }^{1}$ Yamaguchi University, Graduate School of Sciences and Technology for Innovation of Engineering, Faculty of Engineering, 2-16-1 \\ Tokiwadai, Ube-shi, Yamaguchi 755-8611, Japan
}

\begin{abstract}
lon exchange chromatography is a widely used method for purification of all types of biomolecules in current biotechnological downstream processes. Knowledge on the binding behavior of proteins provides valuable insight for understanding the molecular mechanisms of protein interactions in a biological context. However, thermodynamic parameters such as enthalpy and entropy changes that characterize protein adsorption are still unknown. Isothermal titration calorimetry applications in biosciences has gained its merit to study binding of soluble molecules, protein inhibition, conformational changes, reaction kinetics, and protein adsorption. However, in the case of protein adsorption, a lot of complications arise since the usual models used to study protein interactions in solution are no longer valid. This work explains a detailed methodology for the obtention of adsorption enthalpy, entropy and Gibbs energy of protein adsorption, by using ITC together with equilibrium adsorption isotherms.
\end{abstract}

\section{Introduction}

Protein therapeutics have increased dramatically in number and frequency of use. Protein therapeutics already have a significant role in almost every field of medicine, like growth factors, enzymes and antibodies, which are being improved by several approaches. Proteins can be produced by gene expression methods using different expression systems ranging from bacteria to transgenic animals.

One of the most challenging factors on the biomolecule production is its purification from cell culture and most importantly its separation from other similar proteins that are present in the expression systems. Protein chromatography, a technique based on selective adsorption, is able to successfully purify proteins for therapeutic use while following the guidelines required by drug regulatory agencies. Downstream protein processing is now routinely found to be the bottleneck in biopharmaceutical manufacturing because it has not kept pace with upstream production latest improvements. In some cases, the lack of downstream processing capacity can seriously affect the profitability of a new pharmaceutical product and even result in its failure.

Maximum binding capacities and mass transfer properties have been the main focus point of optimizations by the chromatography industry(Müller 2005). However, knowledge on the binding behavior of proteins, liquid-solid interfaces provides valuable insight into the molecular mechanisms that will help the development of new chromatography surfaces, expand existing mechanistic models for adsorption, and stimulate the creation of better downstream purification techniques.

\section{Materials and Methods}

\subsection{Materials}

The selected model stationary phase was Toyopearl Q600C AR (TOSOH Biosciences, Japan) an anionexchanger resin with grafted ligands. Table 1 summarizes the physical properties of the model chromatographic surface. Bovine serum albumin Lot\#037K0765 was purchased from Sigma-Aldrich (USA). It was noticed that commercially protein was not solely composed by BSA in its monomeric isoform but approximately $10 \%$ dimer and $90 \%$ monomer. Before the use of BSA on isothermal titration calorimetry and equilibrium adsorption isotherms experiments, dimers and monomers were separated using a preparative size exclusion chromatography column Superdex Hiload 26/600, 200 pn (GE Healthcare, USA). All salts and buffers used in this work were purchased from Wako (Japan) and dissolved in ultrapure deionized water.

\footnotetext{
Corresponding author: shuichi@yamaguchi-u.ac.jp
} 
Table 1. Summary of the physical properties of the resin used in this study. ${ }^{1}$ information was provided by the manufacturers.

\begin{tabular}{cc}
\hline & Physical properties \\
\hline Pore size of base matrix $[\mathbf{n m}]^{\mathbf{1}}$ & 75 \\
\hline${\text { Particle Size of base matrix }[\boldsymbol{\mu m}]^{\mathbf{1}}}^{\mathbf{1}}$ & 100 \\
\hline Ion Exchange capacity $\left[\mathbf{e q} / \mathbf{L}\right.$ gel] $^{\mathbf{1}}$ & $0.14-0.23$ \\
\hline Ligand attachment chemistry $^{\mathbf{1}}$ & Grafted ligands. 3 $^{\text {rd }}$ \\
& Generation or \\
& Type B \\
\hline
\end{tabular}

\subsection{Adsorption isotherm experiments}

Equilibrium adsorption isotherms were determined with the goal of determine more accurately the amount of protein adsorbed with each injection, explained later in section 2.3. The resins were washed with ultrapure water and later with the respective buffer solution by decantation. The resins were used in a $50 \% \mathrm{resin} / \mathrm{buffer}$ suspension (resin slurry). Equilibrium adsorption experiments were carried out in 96-well acrylic plates. First, the wells were filled with $0.3 \mathrm{~mL}$ different concentration of BSA monomer $\left(\mathrm{BSA}_{\mathrm{m}}\right)$. Initial protein concentration was measured by spectrophotometry at 280 $\mathrm{nm}$ and $300 \mathrm{~nm}$ in a multi-well plate reader PowerWave XS (Biotek, USA). BSA calibration curves were determined in preliminary experiments using 0.2 and 0.3 volume in each well. Then, $20 \mu \mathrm{L}$ of resin slurry $(50 \%$ resin-buffer) was added in each well and stirred inside a temperature controlling chamber for at least 8 hours at 25 ${ }^{\circ} \mathrm{C}$. Preliminary experiments, in the whole range of tested concentrations, showed that equilibrium was reached after only 15 minutes (data not shown). After equilibration, the contents of the wells were transferred to a 96-well filter plate and centrifuged for 3 minutes at 2000 RPM onto a new acrylic 96 -well plate. $0.2 \mathrm{~mL}$ of the filtered solution was transferred to analogous wells in order to keep the solution pathlength constant in every well. The equilibrium concentration was determined using the same method as the initial concentrations. From the equilibrium concentration and initial concentration, the surface concentration could be determined by a mass balance. Equilibrium concentration was plotted against the surface concentration calculated. The experiments were done in duplicate.

\subsection{Isothermal titration calorimetry}

Microcalorimeter used in this work was Nano ITC from TA Instruments (Delaware, USA). It has been already shown that even small $\mathrm{pH}$ differences between the ITC cell and titration solution affects quite significantly the calorimetric results as artifacts in the enthalpy can arise due to buffer protonation effects. Prior to the experiment, $\mathrm{BSA}_{\mathrm{m}}$ solutions were prepared in the respective buffers and exhaustively washed with the respective buffer using AmiconR Ultra-4 10000MW (Milipore Sigma, USA), for $15 \mathrm{~min}$ at $7000 \mathrm{RPM}$. This step was repeated until the conductivity of the buffer solution and protein solution was the same (around 4 to 5 times). Conductivity was checked after every washing step with a portable conductivity meter B-173 Twin Cond acquired from Horiba (Japan).
It the beginning of the calorimetric titration experiment, the reference cell was filled with distilled degassed water (Solution B in fig. 1). The main cell was filled with 1.8 $\mathrm{mL}$ of the slurry resin suspension, i.e., $50 \%(\mathrm{v} / \mathrm{v})$ chromatography resin/buffer (Solution A in fig. 1). The syringe was filled with $\mathrm{BSA}_{\mathrm{m}}$ solution at $20 \mathrm{mg} / \mathrm{mL}$ (Solution $\mathrm{C}$ in fig. 1). The cell was closed and agitated at $300 \mathrm{RPM}$ at $25^{\circ} \mathrm{C}$ After a 5400 seconds' timeout, for the thermal equilibrium can be reached, ten injections of 10 $\mu \mathrm{L}$ were titrated with the $100 \mu \mathrm{L}$ syringe from the ITC equipment. Injections were carried out with a 600 seconds' interval from each other. The temperature fluctuations indicated by the instrument were below $0.0002 \mathrm{~K}$. Both resin slurries and protein solutions were previously degassed in a degassing station at $400 \mathrm{mmHg}$ for 10 minutes (TA Instruments, Delaware, USA).

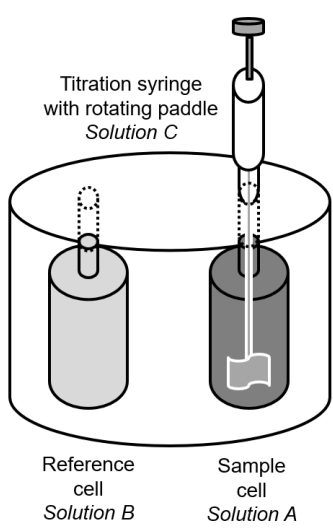

Figure 1. Schematic representation of a typical isothermal titration calorimetry. Solution A: 50\% (v/v) resin/buffer. Solution B: pure water. Solution C: BSA $20 \mathrm{mg} / \mathrm{mL}$.

\section{Results and Discussion}

\subsection{Isothermal titration calorimetry}

The enthalpy change of a system is defined as the sum of the change in internal energy of that system $(\Delta U)$ and the change in the total volume $(\Delta V)$ :

$$
\Delta H=\Delta U+\Delta V
$$

Protein adsorption occurs in virtually incompressible aqueous media $(\Delta V=0)$, this renders $\Delta H$ essencially synonymous with the change in internal energy $(\Delta H=\Delta U)$. By definition:

$$
\Delta U=\Delta Q-\Delta W
$$

where $\Delta Q$ is the heat exchange of the adsorption reaction and $\Delta W$ is the change in work which is approximately zero in molecular reactions $(\Delta W \approx 0)$. Leading to

$$
\Delta H=\Delta Q
$$

ITC directly measures $\Delta Q$, so it can effectively measure the change of enthalpy of protein adsorption. 


\subsection{Infinite dilution and reference state}

The following well known fundamental thermodynamic relation is used in the subsequent derivations already derived by previous authors (Atkins and De Paula 2010; Blaschke et al. 2011):

$$
\left(\frac{\partial\left(\frac{\mu}{T}\right)}{\partial T}\right)_{p, n}=\left(\frac{\partial\left(\frac{G}{T}\right)}{\partial T}\right)_{p, n}=-\frac{H}{T^{2}}
$$

where the subscripts $p$ and $n$ refer to constant pressure and constant composition (closed system). The chemical potential in aqueous solutions $(\mu)$ can be described by

$$
\mu=\mu^{0}+R T \ln \left(C_{i} \gamma_{i}\right)
$$

For infinte dilution $\left(\gamma_{i}=1\right)$, this equation formally reduces to

$$
\mu^{\infty}=\mu^{0}+R T \ln C_{i}^{\infty}
$$

where

$$
C_{i}^{\infty}=\lim _{C_{i} \rightarrow 0} C_{i}
$$

dividing eq. 6 by T results in

$$
\frac{\mu^{\infty}}{T}=\frac{\mu^{0}}{T}+R \ln C_{i}^{\infty}
$$

and differentiating with respect to the respect to the temperature $\mathrm{T}$ under consideration of equation 4 it follows

$$
-\frac{H^{\infty}}{T^{2}}=-\frac{H^{0}}{T^{2}}
$$

and thus,

$$
H^{\infty}=H^{0}
$$

Hence, if the enthalpy obtained calorimetrically was obtained in infinite or close to infinite dilution equals the enthalpy in the reference state

$$
\Delta H^{\infty}=\Delta H^{0}=\Delta H^{a d s}
$$

In practice, this means that in order to use and compare thermodynamic parameters with enthalpy obtained using van't Hoff analysis we should determine the adsorption enthalpy at low concentrations $\left(C_{s} \rightarrow 0\right.$, $C_{l} \rightarrow 0$ ) or take data points at several concentrations and extrapolate the observed $\Delta H^{0}$ to infinite dilution (Werner, Blaschke, and Hasse 2012; Werner, Hackemann, and Hasse 2014).

During the calorimetric measurements, a small signal is observed upon injecting the same solution onto itself. This signal was previously considered to be due to friction, dissipation and other effects like a small mismatch of temperature of the cell and that of the injected solution(Duff, Grubbs, and Howell 2011). Additional contributions to the calorimetric signal can arise from buffer interactions that are independent of the adsorption interactions. These contributions that reflect temperature dependence of buffer ionization as well as of water structure, need to be subtracted from the total measured signal. Considerable attention must be given to the design of adequate control experiments to clarify these contributions(Winzor and Jackson 2006a). The contribution of heat of a protein injection into a resin mixture using ITC can be divided into several origins:

$$
\Delta H^{e x p}=\Delta H^{a d s}+\Delta H^{d i l}+\Delta H^{s o l v}
$$

where $\Delta \mathrm{H}^{\exp }$ is the total enthalpy measured by the ITC. $\Delta H^{0}$ is the enthalpy contribution from the adsorption of the protein into resin. $\Delta \mathrm{H}^{\mathrm{dil}}$ is the contribution of the protein solution dilution heat, measured by titrating protein solution into the same buffer that it was diluted into. $\Delta \mathrm{H}^{\text {solv }}$ is caused by the adsorption of buffer ions and it was determined titrating buffer into the resin slurry. Figure 1 represents typical thermograms obtained for the main experiment and blank trials. So, the blank heat trial were subtracted from the one obtained titrating the protein into the resins, and effective enthalpic contribution from the protein adsorption was obtained.

The first peak of every trial is not shown and it was not used in any calculation due to the well-known phenomenon, named "first-peak anomaly" in previous studies (Werner et al. 2012). Protein injections and all blank trials were done in duplicate.

Adsorption heats were measured directly by ITC, and molar enthalpy of adsorption, $\Delta h^{\text {ads }}$, was then calculated by the following equation:

$$
\Delta h^{\mathrm{ads}}=\Delta H^{\mathrm{ads}} / \Delta n
$$

where $\Delta n$ is the amount of molecule adsorbed upon each injection (mol), which was calculated using a combination of the equilibrium adsorption isotherm and a mass balance.

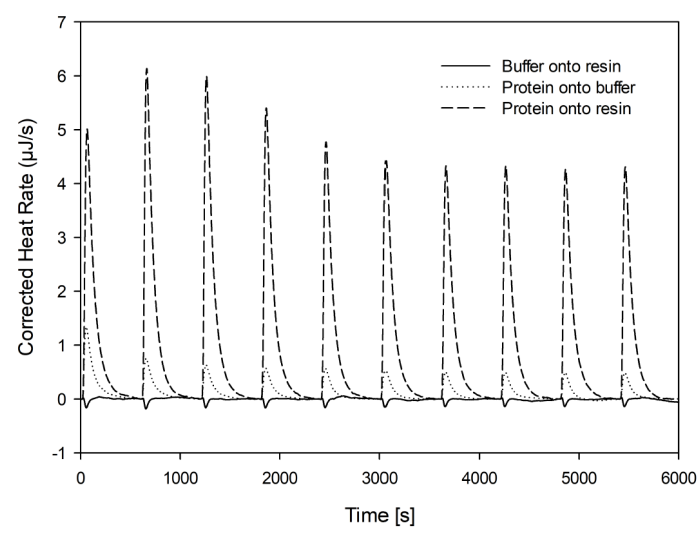

Figure 1. Thermograms obtained titrating BSA monomer at 20 $\mathrm{mg} / \mathrm{mL}$ onto Toyopearl Q600AR and the respective blank trials. Buffer was $10 \mathrm{mM}$ TRIS, $30 \mathrm{mM} \mathrm{NaCl}$ at $\mathrm{pH} 7,25^{\circ} \mathrm{C}$. 


\subsection{Calculation of the amount of molecule adsorbed upon each injection in ITC}

\subsubsection{Equilibrium adsorption isotherm fitting}

Even a polynomial equation with no physical meaning can be used to calculate the amount of molecule adsorbed upon each injection in ITC (Blaschke et al. 2011; Hackemann and Hasse 2017). In this work, the wellknown Langmuir equation was used to relate the equilibrium liquid concentration with surface concentration upon adsorption:

$$
C_{s}=\frac{q_{m} \cdot K \cdot C}{1+K \cdot C}
$$

where $C_{s}$ is the surface concentration (mg of protein $/ \mathrm{mL}$ of resin); $q_{m}$ is the maximum capacity ( $\mathrm{mg}$ of protein $/ \mathrm{mL}$ of resin); $\mathrm{K}$ is distribution coefficient; and $\mathrm{C}$ is liquid concentration after the equilibrium has been reached $(\mathrm{mg} / \mathrm{mL})$. It is important to note that Langmuir equation was not developed for protein adsorption studies and assumes several facts that hold no truth in the case of biomolecule adsorption onto solid phases. Langmuir himself wrote "Considering the nature of the simplifying assumptions made in its derivation it should, of course, not be looked upon as a general equation of the adsorption isotherm" (Rabe, Verdes, and Seeger 2011). Figure 2 represents a typical Langmuir equation fitting.

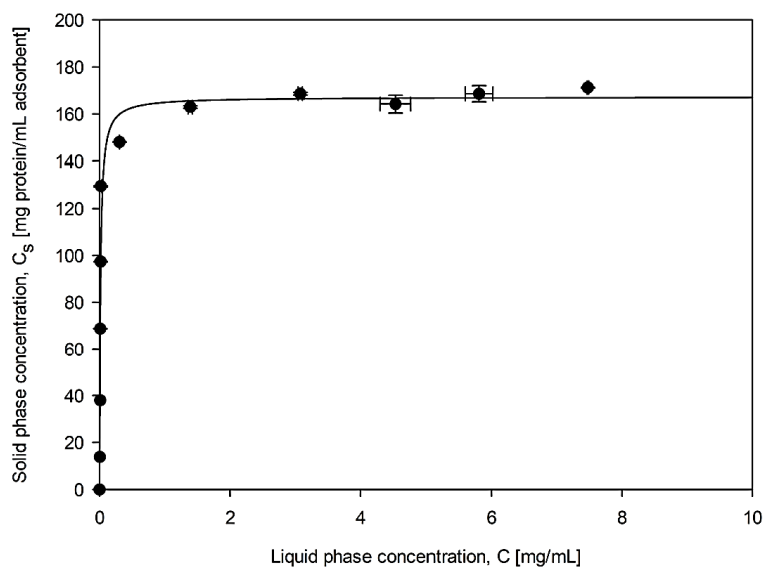

Figure 2. Equilibrium adsorption isotherm obtained at $25^{\circ} \mathrm{C}$. BSA monomer at several concentrations were adsorbed onto Toyopearl Q600AR, 8 hours later the equilibrium liquid phase concentration was determined. Buffer was $10 \mathrm{mM}$ TRIS, 30 $\mathrm{mM} \mathrm{NaCl}$ at $\mathrm{pH} 7,25^{\circ} \mathrm{C}$. Resulting fitting parameters: $q_{m}=$ $167.2, K=72.2$.

\subsubsection{Mass balance}

Since ITC cell and the syringe is a closed system, we may assume preservation of mass in order to calculate solid phase concentration with each injection. In the case of the first injection, a simple mass balance equation can be obtained:

$$
C_{0} V_{0}=C . V_{l}+C_{s} \cdot V_{s}
$$

where $C_{0}$ is the protein concentration in the injection syringe $(\mathrm{mg} / \mathrm{mL}) ; V_{0}$ is the injection volume of each injection; and $V_{l}$ and $V_{s}$ are the volume of liquid and resin inside the ITC cell $(\mathrm{mL})$, respectively. But, with each injection $(i)$, the total liquid volume increases by $V_{0}$. Also, after the first injection, one should take into consideration the amount of protein that was previously adsorbed. Thus, the following mass balance equation can be obtained for any given injection:

$$
\begin{aligned}
C_{s, i-1} V_{s}+C_{i-1}\left[V_{l}\right. & \left.+(i-1) V_{0}\right]+C_{0} V_{0} \\
& =C_{i} \cdot\left(V_{l}+V_{0}\right)+C_{s, i} . V_{s, i}
\end{aligned}
$$

Since $V_{o} \gg V_{l}, V_{0}$ can be neglected in the bracketed terms of the equation. Calculations were made without neglecting $\mathrm{V}_{0}$, and there was no difference between using these terms or not (data not shown). The following system of two equations and two variables can be used from $i=0$ to $i=10$. Solving this system for all injections we have solid and liquid phase concentrations in every injection.

$$
\left\{\begin{array}{c}
C_{s, i-1} V_{s}+C_{i-1} V_{l}+C_{0} V_{0}=C_{i} \cdot V_{l}+C_{s, i} \cdot V_{s} \\
C_{s, i}=\frac{q_{m} \cdot K \cdot C_{i}}{1+K \cdot C_{i}}
\end{array}\right.
$$

Lastly, the number of molecules adsorbed upon each injection (mol), $\Delta n$, can simply be obtained from the difference of surface concentration obtained in consequent injections:

$$
\Delta n=\frac{V_{s}\left(C_{s, i}-C_{s, i-1}\right)}{M_{r}}
$$

where $M_{r}$ is the molecular weight of the protein of study (mg/mol).

\subsubsection{Dependency between $\Delta h^{\text {ads }}$ and $C_{s}$}

Figure 3 shows the resulting $\Delta h^{\text {ads }}$ from each injection and the surface concentration at which were obtained. As explained in section 3.2, the first peak is ignored due to observable differences. We believe this happens due to two reasons: (1) diffusion of protein from the syringe in the equilibration step, and (2) possible air micro bubbles contained in the end of the syringe that are released in first injection.

As explained in section 3.2 , in order to calculate following thermodynamic parameters by the generally know Gibbs-Helmholtz equation:

$$
\Delta G^{a d s}=\Delta H^{a d s}-T \Delta S^{a d s}
$$

where $\Delta G^{a d s}, \Delta S^{a d s}$ and $T$ represent the change on the standard free Gibbs energy upon adsorption, the change on the standard entropy and the absolute temperature, respectively. In order to use this relation, that assumes infinite dilution conditions, it is necessary to extrapolate the $\Delta H^{a d s}$ to the intersect $\left(C_{s} \rightarrow 0\right)$. Figure 3 represent 
two slightly different examples. At $\mathrm{pH}$ 8.5, no extrapolation is needed because $\Delta h^{\text {ads }}$ is constant throughout the experiment (confirming infinite dilution conditions). At $\mathrm{pH} 7$, the effective enthalpy was assumed to be the one determined with injection 2 and 3, while we can assume infinite dilution conditions.

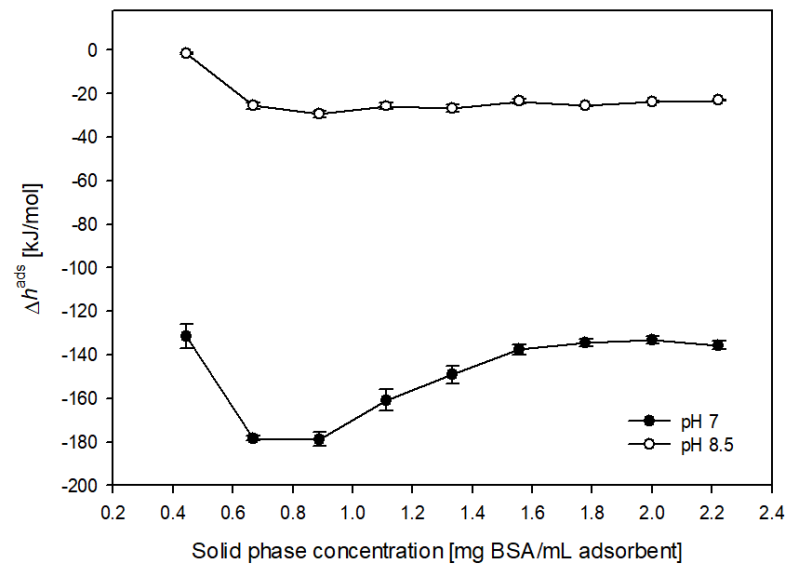

Figure 3. BSA adsorption onto toyopearl Q600C-AR. $\Delta h^{\text {ads }}$ obtained from isothermal titration calorimetry at each injection. Buffer was $10 \mathrm{mM}$ TRIS, $30 \mathrm{mM} \mathrm{NaCl}(\bullet) \mathrm{pH} 7$ and (O) $\mathrm{pH}$ 8.5 .

\subsubsection{Gibbs energy determination}

For a reaction to occur spontaneously, like adsorption, the Gibbs free energy of the initial state $\left(G_{\text {initial }}\right.$ ) is necessarily higher than that $G_{\text {final }}$ of the state towards which the reaction is heading. In an equation form:

$$
\Delta G=G_{\text {final }}-G_{\text {initial }} \leq 0
$$

where $\Delta G$ is the amount of energy available for chemically driven work. The change in Gibbs free energy differs from the enthalpy change $(\Delta H)$ in that it does not include contributions to energy arising from volume or pressure changes, nor does it include the entropic contributions $(T \Delta S)$.

In liquid chromatography of biomolecules, temperature, pressure and chemical potential of the solvent usually remain constant. Under these conditions, Gibbs energy of the adsorbing protein $\left(G_{i}\right)$ is related to its molar concentration $\left(C_{i}\right)$ and protein activity coefficient $\left(\gamma_{i}\right)$ by the following equation(Winzor and Jackson 2006b):

$$
\Delta G_{i}=\Delta G_{i}^{0}+R T \ln \left(C_{i} \gamma_{i}\right)
$$

where $G_{i}^{0}$ is the standard free energy defined under the constrains of constant temperature and solvent chemical potential (reference state); $\mathrm{R}$ is the gas constant and $\mathrm{T}$ is the absolute temperature.

A simple protein adsorption reaction can be written as $L \leftrightarrows S$ where $L$ and $S$ is the free protein in liquid phase and solid phase, respectively. The free energy change, $\Delta G=G_{S}-G_{L}$ can be written as:

$$
\Delta G=\Delta G^{0}+R T \ln \left(\frac{C_{S}}{C_{l}} \frac{\gamma_{s}}{\gamma_{l}}\right)
$$

In practice the ratio of activity coefficients may be taken as unity $\left(\gamma_{s} / \gamma_{l} \approx 1\right)$ if we assume infinite dilution conditions to approach thermodynamic ideality. In equilibrium, $\Delta G=0$ in equation 21 , the standard free energy change under conditions of thermodynamic ideality is related to the equilibrium concentrations of participating species by the expression

$$
\Delta G^{0}=-R T \ln \left(\frac{C_{S}{ }^{e}}{C_{l}^{e}}\right)
$$

where the superscripts $e$ are used to identify concentrations at adsorption chemical equilibrium. Since the distribution coefficient $K$ is by definition the ratio of equilibrium concentrations, equation 21 can be expressed as

$$
\Delta G^{a d s}=-R T \ln K
$$

On the other hand, if described with the dissociation ratio $K_{d}=C_{l}{ }^{e} / C_{s}{ }^{e}$, the relationship becomes

$$
\Delta G^{a d s}=R T \ln K_{d}
$$

Distribution coefficient $K$ can be determined experimentally via several methods, such as, isocratic elution mode, application of $G H-I_{R}$ model to linear gradient elution mode(Yamamoto 2005), and as an example stated in this short study, from equilibrium adsorption isotherms fitting. Consequently, $\Delta G^{\text {ads }}$ of $\mathrm{BSA}_{\mathrm{m}}$ adsorption onto Toyopearl Q600C-AR was determined and shown in table 2 .

\subsubsection{Determination of the change on adsorption entropy}

Although $\Delta G^{a d s}$ is enough to define the energetics of protein adsorption, there are considerable advantages in its breakdown into enthalpic ( $\Delta H^{a d s}$ ) and entropic contributions $\left(\Delta S^{a d s}\right)$ that can be represented by equation 18 .

The behavior of proteins in adsorption is a net result of various types of interactions already mentioned in previous section. If the entropy term contributes to $\Delta G$ in such a way that it becomes negative, the process is called entropy driven; if exothermic enthalpy values are dominant, leading to a negative $\Delta G^{0}$, the process is considered enthalpically driven.

From enthalpic contributions, we can gain molecular insight on the nature of the predominant noncovalent interactions responsible for adsorption. For example, hydrogen bonding and ionic interactions are generally enthalpically driven (negative $\Delta H^{a d s}$ ) whereas hydrophobic interaction derive their strength from a positive $\Delta S^{\text {ads }}$ that outweighs the consequences of any enthalpic contributions. 
Currently there is no list or detailed separation between enthalpically driven events from entropically driven ones due to their inseparable nature. The enthalpy change depends on the entropy change and vice versa.

All things considered, $T \Delta S^{\text {ads }}$ can be directly obtained using equation (18) if $\Delta H^{a d s}$ and $\Delta G^{a d s}$ were obtained in the same experimental conditions assuming infinite dilution. Resulting in the calculation of the following thermodynamic parameters shown in table 2 .

Table 2. Thermodynamic parameters for $\mathrm{BSA}_{\mathrm{m}}$ adsorption onto Toyopearl Q600C-AR determined with ITC together with equilibrium adsorption isotherms. $30 \mathrm{mM}$ TRIS, $30 \mathrm{mM} \mathrm{NaCl}$ $\mathrm{pH} 7$, at $25^{\circ} \mathrm{C}$.

\begin{tabular}{clr}
\hline$\Delta \boldsymbol{H}^{\text {ads }}$ & $\mathrm{kJ} / \mathrm{mol}$ & -178.6 \\
\hline $\boldsymbol{K}$ & $\mathrm{mL}$ liquid phase $/ \mathrm{mL}$ resin & 72.2 \\
\hline$\Delta \boldsymbol{G}^{\text {ads }}$ & $\mathrm{kJ} / \mathrm{mol}$ & -10.6 \\
\hline$\Delta \boldsymbol{S}^{\text {ads }}$ & $\mathrm{kJ} / \mathrm{mol} / \mathrm{T}$ & 0.56 \\
\hline
\end{tabular}

\section{Conclusion}

The adsorption enthalpy of the binding/partition process of proteins with ionic exchange are measured by ITC and reported in this study. $\mathrm{K}$ can be calculated via several methods, in this case it was calculated by a Langmuir equation fited to the equilibrium binding isotherm.

One of the drawbacks of ITC is that the adsorption process is divided at least five sequential processes, however, ITC has only capacity of showing the overall heat of adsorption and cannot discern different subprocesses of adsorption in a chronological manner. This means that the researcher should be careful not to conclude that the major contributions are the only contributions for adsorption, i.e., just because $\Delta H^{a d s}$ is negative does not mean that entropy is irrelevant for adsorption.

The interaction mechanism of proteins with ion exchange chromatography resins can be studied with ITC and this technique is advantageous in the study of protein adsorption.

\section{References}

Atkins, P. W. and Julio De Paula; 2010, Atkins' Physical Chemistry, Oxford University Press (2019)

Blaschke, T., J. Varon, A. Werner, and H. Hasse; "Microcalorimetric Study of the Adsorption of PEGylated Lysozyme on a Strong Cation Exchange Resin," Journal of Chromatography A, 1218(29), 4720-26 (2019)

Duff, M. R., J. Grubbs, and E. E. Howell; "Isothermal Titration Calorimetry for Measuring MacromoleculeLigand Affinity," J. Vis. Exp., 55, https://doi.org/10.3791/2796 (2011)

Hackemann, E. and H. Hasse; "Influence of Mixed Electrolytes and $\mathrm{PH}$ on Adsorption of Bovine Serum Albumin in Hydrophobic Interaction Chromatography," Journal of Chromatography A, 1521, 73-79 (2017)
Müller, E.; "Properties and Characterization of High Capacity Resins for Biochromatography," Chemical Engineering \& Technology, 28(11), 1295-1305 (2015)

Rabe, M., D. Verdes, and S. Seeger; "Understanding Protein Adsorption Phenomena at Solid Surfaces," Advances in Colloid and Interface Science, 162(1-2), 87$106(2011)$

Werner, A., T. Blaschke, and H. Hasse; "Microcalorimetric Study of the Adsorption of PEGylated Lysozyme and PEG on a Mildly Hydrophobic Resin: Influence of Ammonium Sulfate," Langmuir, 28(31), 11376-11383 (2012)

Werner, A., E. Hackemann, and H. Hasse; “Temperature Dependence of Adsorption of PEGylated Lysozyme and Pure Polyethylene Glycol on a Hydrophobic Resin: Comparison of Isothermal Titration Calorimetry and van't Hoff Data," Journal of Chromatography A, 1356, 188 196 (2014)

Winzor, D. J. and C. M. Jackson; "Interpretation of the Temperature Dependence of Equilibrium and Rate Constants," JMR, 19, 389-407 (2006)

Yamamoto, S.; "Electrostatic Interaction Chromatography Process for Protein Separations: Impact of Engineering Analysis of Biorecognition Mechanism on Process Optimization," Chemical Engineering and Technology, 28(11), 1387-1393 (2011) 